How to reference this article Kornacka, B. (2020). I personaggi maschili ne L'arte della gioia di Goliarda Sapienza. Italica Wratislaviensia, 11(2), 97-117.

DOI: http://dx.doi.org/10.15804/IW.2020.11.2.6

\author{
Barbara Kornacka \\ Uniwersytet im. Adama Mickiewicza w Poznaniu, Polonia \\ kornacka@amu.edu.pl \\ ORCID ID: 0000-0001-7143-404X
}

\title{
I PERSONAGgI MASCHILI NE L'ARTE DELLA GIOIA DI GOLIARDA SAPIENZA
}

\section{THE MALE CHARACTERS IN L'ARTE DELLA GIOIA BY GOLIARDA SAPIENZA}

\begin{abstract}
The aim of the paper is to analyse, using the structuralist approach, the structure of the male characters in L'arte della gioia by Goliarda Sapienza and the meaning - universal or local, symbolic or real-attributed by the writer to them, through the example of six male figures. This approach allows us to delve into the literary text, leaving the biographical, historical, and ideological context outside of the analysis, focusing on internal dynamics that are purely literary. Modesta's father and her husband, as universal figures of a patriarchal system, illustrate and symbolise the subversion of its laws by Modesta. Modesta's lovers, Carmino and Carlo, are models of real accomplishments of important male roles (father, husband, lover) whose representation is limited by geopolitical stereotypes. Pietro, Modesta's loyal gabellotto, embodies local, Sicilian relationships and, as a result, he symbolises the persistence of archaic laws of Sicilian society. With Prando, Modesta's son, Sapienza realistically illustrates the passage from adolescence to masculinity by showing some universal rules that occur in this transition. The structure of the male characters turns out to be geometric and very clear and is proof of the writer's great literary awareness and writing skills.
\end{abstract}

Keywords: Goliarda Sapienza, Arte della gioia, masculinity, universality, sicilianity 
Coliarda Sapienza (1924-1996), scrittrice, attrice, poetessa e drammaturga catanese, quasi coetanea di Italo Calvino (1923-1985) e di Oriana Fallaci (1929-2006), è protagonista della più clamorosa e sorprendente scoperta letteraria degli ultimi quindici anni ${ }^{1}$. Poco conosciuta in vita, presto dimenticata dopo la morte, nell'arco di una quindicina di anni, dal 2005, anno di uscita e di eccezionale successo de L'arte della gioia ${ }^{2}$ in Francia ${ }^{3}$, "la princesse hérétique" - come è chiamata la scrittrice da "Le Monde" (de Ceccatty, 2005) - diventa oggetto di interesse sempre crescente di critici e studiosi di letteratura, argomento di convegni, ricerche e colloqui, di alcune monografie ${ }^{4}$ e numerosissimi articoli e comunicazionis ${ }^{5}$.

La sua lunga e dapprima stentata inclusione nella letteratura cosiddetta canonica inizia con il volume di Giovanna Providenti (2010): uno studio principalmente biografico, in cui la studiosa, tuttavia, non solo ricostruisce minuziosamente e appassionatamente gli elementi della vita professionale e privata della scrittrice (oramai ben noti), ricollegandoli abilmente con Modesta, ma riflette anche sulla storia del femminismo in Italia e sull'apparente libertà della donna. Solo un anno più tardi esce un'importante miscellanea a cura di Monica Farnetti (2011), la quale, oltre alla presentazione - con una serie di memorie - della storia della riscoperta di Sapienza in Italia, offre alcune prime analisi dei romanzi della scrittrice tra cui anche de L'arte della gioia. Meritano un accenno i contributi della Farnetti (sulla simbologia dei matricidi all'insegna della critica femminista) e di Laura Fortini (sulla critica del patriarcato nel testo di Sapienza attraverso la figura paterna e la conseguente distanza

1 Della riscoperta di Sapienza che inizia con il romanzo L'arte della gioia parlano nei rispettivi contributi Manuela Vigorita (Vigorita, 2011, pp. 20-24) e Loredana Rotondo (Rotondo, 2011, pp. 26-28).

2 Edizione di riferimento: Sapienza, 2014.

3 La prima edizione francese del romanzo, con la traduzione di Nathalie Castagné, è stata pubblicata presso la casa editrice Éditions Viviane Hamy. Nel 2018 per i tipi di Wydawnictwo Kobiece il romanzo è uscito in Polonia tradotto da Tomasz Kwiecień.

4 Cf. Bazzoni, 2018; Bazzoni, Bond \& Wehling-Giorgi, 2016; Providenti, 2010; Providenti, 2016; Rizzarelli, 2018; Scarfone 2018; Trevisan, 2016.

5 Una vasta bibliografia è fornita da Alessandra Trevisan, 2016. 
del romanzo dal modello del romanzo ufficiale). L'anno successivo vede la luce un'altra importante miscellanea curata dalla Providenti (2012) il cui filo conduttore è l'essere pirandelliano ovvero l'autenticità della scrittrice e del suo oeuvre. Le tre parti del volume sono rispettivamente dedicate a L'arte della gioia, alle relazioni tra la vita di Sapienza e la sua opera e al tema dell'ambivalenza nella sua espressione artistica. Come frutto di un importante convegno londinese (2013) esce il volume Goliarda Sapienza in Context (2016) curato da Alberica Bazzoni, Emma Bond e Katrin Wehling-Giorgi, nel quale si riflette sull'opera della scrittrice - come indica il titolo stesso del volume - nel contesto della cultura italiana ed europea. I contributi della prima parte riesaminano e approfondiscono la questione dell'intreccio tra vita e opera della scrittrice, tra la sua identità e l'espressione artistica. Gli articoli della seconda parte offrono uno studio delle influenze e delle intertestualità che intercorrono tra Sapienza e altri autori internazionali. La terza parte invece è finalizzata al reinserimento di Sapienza nel contesto della tradizione letteraria italiana. Di particolare rilievo è qui lo studio della Fortini Beyond the Canon: Goliarda Sapienza and Twentieth Century Italian Literary Tradition, in cui la studiosa propone di collocare Sapienza tra le altre scrittrici italiane sperimentali e innovative del Novecento, le quali si vogliono vedere ai margini del canone. Fortini suggerisce di applicare la categoria del romanzo del divenire, ovvero il Bildungsroman delle donne $^{6}$. L'ultima parte del volume raccoglie gli articoli che offrono una riflessione sugli spazi, concettuali e fisici, presenti nell'opera di Sapienza in riferimento anche alle altre scrittrici del Novecento come Dacia Maraini o Elena Ferrante. Sempre nel 2016, ad approfondire la ricerca su Goliarda Sapienza, esce una ricca e completa monografia di Alessandra Trevisan Goliarda Sapienza. Una voce intertestuale. La studiosa vi ripercorre la vita e le varie manifestazioni (romanzi, poesia, teatro) dell'espressione artistica di Sapienza rintracciandovi sempre la stessa voce che si afferma e unisce l'esistenza e l'arte. Più recentemente, nel 2018 appaiono tre monografie sulla scrittrice. Nello studio intitolato Writing for Freedom. Body, Identity and Power in Goliarda Sapienza's

${ }^{6}$ Il concetto del romanzo del divenire è stato presentato in Fortini \& Bono, 2007. 
Narrative Alberica Bazzoni ripercorre la narrativa di Sapienza delineando la sua poetica, incentrata - secondo la principale tesi del volume sulla ricerca della libertà ideale. Maria Rizzarelli nella sua monografia Goliarda Sapienza. Gli spazi della libertà, il tempo della gioia organizza la ricerca attorno all'autobiografismo della scrittrice, definendolo queer, dal momento che vi si assiste a una continua scomposizione dei limiti del genre (testo) e del genere (vita). La studiosa legge tutta l'opera di Sapienza come un macrotesto unito dalla costellazione tematica spaziocorpo-identità. L'autrice della terza monografia (Gloria Scarfone, Goliarda Sapienza. Un'autrice ai margini del sistema letterario) rinuncia alla prospettiva dei Gender Studies o dei Queer Studies e si fissa invece l'obiettivo di “storicizzare l'opera di Sapienza - soprattutto L'arte della gioia - problematizzandola a livello di genere, individuandone le fonti e contestualizzandola all'interno del sistema letterario del secondo Novecento" (Scarfone, 2018, p. 13).

Più studiato e analizzato oramai da molteplici prospettive è il romanzo che contribuì alla riscoperta della scrittrice, L'arte della gioia ${ }^{7}$. Sono numerosi gli studi validi e di grande interesse, tra cui si menziona in questa sede a titolo di mero esempio quello di Lara Michelacci (2015) che riflette sul genere del romanzo nel contesto della storia di questo genere letterario. Nel romanzo ibrido di Sapienza vi sono anche gli elementi del romanzo storico, in cui a confrontarsi con la Storia è una storia individuale. Il concetto della Storia come movimento lineare (anni) e come movimento circolare (stagioni), entrambi presenti nei contenuti e nelle strutture narrative del romanzo, è invece il fulcro dell'interessantissima riflessione di Alberica Bazzoni (2012).

Il romanzo, spunto anche delle presenti riflessioni, parla di Modesta, una paesana siciliana che, grazie al suo coraggio, all'intelligenza, alla sua forza di carattere nonché talvolta all'astuzia e crudeltà, diventa una potente principessa. La storia della sua emancipazione sociale - una cornice questa, tuttavia, molto generica che contiene numerosi temi, motivi e offre molte possibilità interpretative - è al contempo cronaca

7 Si veda almeno la bibliografia di cui sopra (nota 5), ricca ma non esaustiva, non completa degli studi apparsi tra il 2016 e il 2020. 
della ricerca e del raggiungimento della liberazione dai limiti sociali e culturali, dai tabù, dagli stereotipi, dagli usi e pregiudizi da parte di una donna, sullo sfondo di circa settant'anni di storia italiana e sicilia$\mathrm{na}^{8}$. Con il romanzo a cui dedica otto anni della sua vita (1968-1976), oramai da anni lontana dalla sua terra ${ }^{9}$, la scrittrice torna al suo vissuto personale: alla sua esperienza culturale, linguistica, sociale e materiale della terra siciliana per la quale "nutre un'attrazione-repulsione molto forte" (Trevisan, 2016, p. 30). La Sicilia è per Sapienza "una terra in cui perdurano i pregiudizi e omertà, ma che resta incarnata nel suo corpo e soprattutto nella sua lingua, più volte repressa eppure viva, attestazione della provenienza ma soprattutto dell'identità e dell'eloquenza scrittoria" (Trevisan, 2016, p. 30). Tuttavia, ne L'arte della gioia l'isola natia assume, inoltre, caratteri di universalità. Come nota Laura Fortini:

L'arte della gioia non è il romanzo siciliano se non nella dimensione che fa dell'isola mondo, senza che con ciò si neghi la precisa localizzazione geografica della vicenda: che si svolge interamente, o quasi, in Sicilia, e che alla lingua madre siciliana si volge per alternare l'italiano medio con la lingua dell'esperienza, quella dell'iniziazione erotica ma soprattutto quella del rapporto con la madre [...]. (Fortini, 2011, pp. 116-117)

La maggior parte degli studi sull'opera di Goliarda Sapienza rappresentano l'approccio femminista o/e quello degli studi di genere, spesso abbinato alla prospettiva biografica. Nel presente contributo si preferisce invece un approccio critico meno collimante con le tematiche identitarie e corporali delle opere della scrittrice e sicuramente meno fresco, ovvero l'approccio strutturalista, il quale consente di addentrarsi nel testo letterario lasciando al di fuori dell'analisi, contrariamente all'orientamento biografico più frequentemente adottato dalla critica, il contesto biografico, storico e ideologico, focalizzandosi sulle dinamiche interne puramente letterarie. Vista un'ipotetica ampiezza di tale esame dell'intera opera ci si prefigge in questa sede l'obiettivo di delineare

8 Cf. Bazzoni, 2012; pp. 33-52; Michelacci, 2015, pp. 1-15.

9 Nel 1941, a soli diciassette anni la scrittrice si trasferisce a Roma per studiare presso l'Accademia nazionale d'arte drammatica. Da quel momento vive fuori dall'isola. 
e descrivere il sistema dei personaggi maschili, che forma, all'avviso di chi scrive, una struttura molto ponderata, intelaiata per mettere in rilievo il punto centrale del romanzo, ovvero Modesta, ma anche, cosa non meno importante, convergente con la tessitura dell'intero romanzo a più livelli che si stende tra le valenze simboliche e reali, locali e universali. Il ricorso agli inevitabili termini derivanti dalla critica di genere quali la mascolinità o dalla critica femminista (cultura patriarcale) è puramente strumentale e soggetto alla ricerca strutturale.

Si vuole, quindi, riflettere su come la scrittrice, costruendo i personaggi maschili, si muova abilmente, da un lato, tra il simbolico e il reale, dall'altro, tra il locale (siciliano) e l'universale. A tal fine si analizzeranno le sei figure maschili più importanti ed eloquenti il cui ruolo non si limita alla pura funzionalità nelle dinamiche favolistiche del testo bensì nell'economia del romanzo è quello di veicolare precisi messaggi della scrittrice.

In primo luogo, l'analisi verterà sulle figure emblematiche del sistema patriarcale ovvero sul padre (anonimo) e sul marito (Ippolito). In secondo luogo, si esamineranno i due più rilevanti amanti maschili di Modesta: Carmine (siciliano) e Carlo (milanese), in una specie di rapporto binario e antitetico, riconducibile in parte all'ottica degli stereotipi di genere vigenti nella geopolitica italiana nel Novecento (Dell'Agnese, 2007, p. 18). In seguito, si vedrà la specificità ambigua della mascolinità siciliana di stampo mafioso con l'esempio di Pietro e del suo rapporto di leale servitù alla padrona, Modesta. In ultimo luogo, il sesto personaggio analizzato sarà Prando, figlio di Modesta e Carmine, rappresentato nel corso del suo passaggio dall'età adolescente a quella adulta, che, al contempo, è un passaggio dai valori vigenti nell'ambiente familiare e insegnati dalla madre (libertà, parità, democrazia) a quelli previsti per la mascolinità in una società patriarcale (prepotenza, dominio $)^{10}$.

10 Circa i termini "maschilità" e "mascolinità" si precisa che il primo, il cui significato secondo il dizionario è "una partecipazione a caratteri fisiologicamente o tradizionalmente propri del maschio" (Devoto \& Oli, 1990) "comunica una nozione del maschile più neutra e quasi paradigmatica" (De Biasio, 2010, p. 11) il secondo invece essendo una "qualità definita dalla presenza di caratteri accentuatamente maschili" (Devoto \& Oli, 1990) denota caratteri qualificativi quali ad esempio forza, audacia 


\section{IL PADRE E IL MARITO}

Il padre e il marito sono figure emblematiche, egemoniche e dominanti dell'ordine simbolico e dell'organizzazione patriarcale della cultura nonché simboli del potere e della Legge nelle società tradizionali. Nell'intero romanzo ${ }^{11}$ si fa costantemente rinvio alle diverse forme in cui si manifesta il potere patriarcale ${ }^{12}$ di cui è vittima anche la protagonista, stuprata da bambina - immagine più che illustrativa - dal padre. Quest'ultima, tuttavia, nel corso della sua vita impara e attua le strategie per opporsi efficacemente alla potestà patriarcale. Attraverso la sua eccellente "personaggia" rative, rinvii intertestuali o altre soluzioni letterarie, Goliarda Sapienza affronta e annienta l'ordine simbolico patriarcale che assume molteplici volti, anche quello della tradizione letteraria canonica (cf. Fortini, 2011, pp. 119-126). A tal fine contribuisce tra l'altro la marginale apparizione del padre e del marito nonché la loro bassa e screditata posizione nella gerarchia dei soggetti del romanzo.

Il padre di Modesta appare soltanto in una delle prime scene del libro e si presenta inizialmente come un uomo bello e attraente: "alto e robusto" con i denti bianchi e gli occhi azzurri e con "il petto e i fianchi robusti senza un filo di grasso" (p. 11). Il suo corpo maschile, il suo atteggiamento e la sua "voce morbida come la pelle della giacca" destano nel primo momento un vivo interesse nella bambina. A sentire il padre Modesta comincia a nutrire la speranza di poter finalmente parlare: "Mi piaceva parlare e ridere con lui. Né la mamma né Tina parlavano mai.

e così via (De Biasio, 2010, p. 11). Ne consegue che il termine di maschilità preserva una certa neutralità storica, culturale e sociale. Invece quello di mascolinità, che deriva dall'attribuzione al maschio di certe qualità prestabilite, convenute, si configura come un costrutto culturale soggetto al discorso (De Biasio, 2010, p. 11). Nella presente analisi userò entrambi i termini a seconda del caso, della necessità e del significato che accentuerò.

${ }^{11}$ L'azione si svolge interamente in Sicilia, a Catania e nei suoi dintorni. Inizia con i primi ricordi d'infanzia della protagonista nata il 1 gennaio del 1900 e finisce negli anni Settanta.

12 Per approfondire si veda Bazzoni, 2018, pp. 123-136.

13 Cf. Mazzanti, Neonato, \& Sarasini, 2016. 
Ora con lui avrei parlato, invece di andare a sfogarmi col vento come avevo fatto sempre" (p. 11). Con l'apparizione di quel bel padre nella sua vita nasce il desiderio di usare la parola e la voce, letteralmente ma soprattutto nel senso metaforico, ovvero di avere il potere e i diritti, di essere come lui, come un uomo. Il desiderio che tornerà più volte nel corso del romanzo e che Modesta senza esitazioni metterà in atto proprio a partire da questo primo momento quando appoggia e asseconda i gesti brutali e aggressivi del padre nei confronti della madre che poi ucciderà lei stessa ${ }^{14}$. Intanto, quel padre prestante e promettente, nell'atto di una violenza incestuosa compiuto sulla bambina, rivela nella stessa scena la sua vera natura ingannevole e traditrice. Modesta che "doveva essere forte come lui" (p. 13) prende allora la sua prima decisione da donna ("Io prima ero una bambina, ma ora sono diventata femmina e devo stare attenta") e sarà quella di annullare la madre, modello rifiutato di femminilità sottomessa e muta, nonché il padre, di cui non si ritrova il corpo e di cui non si saprà più niente nel romanzo. La sua figura è stata cancellata. Appiccando il fuoco - elemento che distrugge e purifica al contempo - alla casa, dove suo padre dorme, Modesta lo elimina dalla sua vita e simbolicamente spodesta la figura paterna, dando così inizio allo smontare, che si attuerà continuamente nelle pagine del romanzo, dell'ordine simbolico patriarcale.

Il piano di ribaltare le sue gerarchie prevede anche lo spodestamento della figura molto potente e rilevante del sistema patriarcale, ovvero quella del marito, il cui ruolo coincide tradizionalmente con l'esercizio del potere, nonché con il controllo derivante dalla posizione di chi possiede sia la donna sia i beni materiali. Il matrimonio di Modesta sembra una parodiagrottesca, quasi carnevalesca - visto il rovesciamento dei ruoli - di tale sistema poiché Ippolito, suo marito e figlio della principessa Gaia, chiamato da tutti "la cosa", era un giovane uomo "tozzo e grasso con una testa tonda" (p. 84), chiuso in una stanza del palazzo, con le mani legate, che non sapeva parlare - anche l'incapacità vocale può essere letta in chiave simbolica - perché era affetto da mongolismo,

${ }^{14}$ Sulla valenza simbolica dei tre matricidi vedi Farnetti, 2011, pp. 89-100; Di Rollo, 2016, pp. 33-45; Michelacci, 2015, pp. 1-15. 
infermità per cui venne rifiutato perfino dalla madre che non lo vide più dal giorno della nascita. Da sempre circondato da un alone di paura e ribrezzo, a Modesta fa semplicemente pensare a Tina, sua sorella mongoloide. Con astuzia Modesta escogita quindi un progetto di matrimonio "anche se malato, uomo era, e principe" (p. 94) pensa la ragazza - che le dà non solo l'alta posizione sociale, la ricchezza ma anche, al contrario di quanto avviene con le donne sposate, l'indipendenza e molta libertà. Mentre quindi la figura del padre, del patriarca si vorrebbe dire, qui stupratore incestuoso e pedofilo, scompare nel nulla forse ridotta in cenere, la figura del marito, secondo padrone della donna, è un disabile mentale innocuo, senza capacità di intendere e volere, senza abilità della parola, del tutto disarmato e sottomesso.

Entrambe le figure del potere patriarcale sono quindi dotate di valenza simbolica universale. La loro presenza nel romanzo è tuttavia controbilanciata da un'altra coppia di protagonisti che costituiscono, almeno in parte, progetti di una reale attuazione, più armoniosa e più collaborativa dei suddetti ruoli maschili.

\section{AMANTI}

Si pensa qui a Carmine e a Carlo, due uomini amati da Modesta e amanti molto importanti nella sua vita. Carmine è padre di Beatrice e di Prando, figlio di Modesta. Anch'egli può essere letto come figura paterna, data la sua età e il fatto che era amante di madre Leonora, una delle figure materne per la protagonista, nonché madre biologica di Beatrice. D'altronde Modesta a un certo punto confronta la coppia Carmine-Leonora pensando: "Anche Carmine mi aveva fatto paura, ma non allo stesso modo di madre Leonora" (p. 105). Nonostante le accuse di prepotenza e autorevolezza, pronunciate da Matteo, suo figlio adulto, Carmine nei confronti della protagonista manifesta alcuni aspetti paterni positivi: è protettivo, premuroso e discreto, le insegna come gestire i poderi dei Brandiforti e come fare la padrona. In più la istruisce - e qui entriamo nell'ambito del suo ruolo principale nel romanzo, quello dell'amante nell' ars amandi. Carlo invece è marito di Beatrice e sarebbe forse il modello di marito ideale - rispettoso, attento, delicato, intelligente, leale, 
premuroso - se non ci fosse il suo rapporto con la sessualità, sia con quella dell'uomo sia con quella della partner.

Entrambi gli amanti rappresentano due modelli opposti del rapporto che l'uomo instaura con il proprio corpo, la propria sessualità e quella della donna. Carmine, uomo di una certa età, amministratore delle terre e gabellotto della famiglia Brandiforti, nel vivere la sua sessualità sembra essere libero dai suggerimenti e dalle imposizioni della cultura e della tradizione che stanno alla base dell'ordine simbolico patriarcale. Nei rapporti sessuali Carmine è lungi da considerare il suo corpo come strumento del potere nell'ottica dell'immaginario maschile (cf. Ciccone, 2009, p. 76) e se stesso l'unico soggetto del piacere (Ciccone, 2009, p. 85). Dai primi incontri con Modesta, Carmine riconosce il desiderio e la soggettività della donna e il suo diritto al piacere sessuale. "Scusami, piccirida, di 'sta fretta, è che da tanto ti volevo e tu niente sai fare. Piano piano, col tempo, $t$ 'insegno a venire anche te. Niente vi insegnano le vostre madri, e tocca all'uomo poi..." (p. 109). Nelle parole di Carmine citate sopra risuona tuttavia un'accusa nei confronti delle donne stesse che istruite e abituate alla sottomissione all'uomo nelle società patriarcale, non hanno una tradizione transgenerazionale di preparazione a una soddisfacente vita erotica. Per poter condividere con Modesta l'esperienza amorosa le spiega, da amante-padre ("Vedi, figghia", p. 109), i meandri della tecnica erotica ricorrendo alla metafora dello strumento: "La verità è che quando trovi la donna giusta o l'uomo giusto, allora è di dovere intendersi. Il corpo uno strumento delicato è, più di una chitarra, e più lo studi e più l'accordi all'altro, più diventa perfetto il suono e forte il piacere. Ma tu ti devi aiutare e aiutarmi" (p. 110). Quella di Carmine è una sessualità maschile moderna: aperta alla complicità con la donna, sessualità maschile che si realizza nella complementarietà e nella collaborazione con la donna. La maschilità di Carmine proposta nel romanzo da Sapienza corrisponde a quanto, molti anni più tardi, propone a questo proposito Stefano Ciccone:

L'incontro con un altro desiderio e quindi con la libertà e la soggettività femminile rende infatti possibile una nuova esperienza del corpo maschile in cui la scoperta di un limite (l'esistenza di un'altra soggettività e di un al- 
tro desiderio) può arricchire la mia vita sessuale e affettiva. Mi rivela nuove potenzialità del mio corpo, mi fa vedere me stesso non solo come soggetto desiderante ma anche come oggetto di un desiderio altro che non è semplice specchio del mio [...]. Il riconoscimento dell'autonomia del desiderio dell'altra permette di riscoprire il corpo maschile e superare la scissione tra il proprio sesso e la propria persona intesa come complesso di sensazioni e di sentimenti, tra il proprio piacere e quello della donna. (Ciccone, 2009, p. 86)

Il modello della sessualità maschile antitetico è quello rappresentato da Carlo che, nonostante la sua ottima formazione intellettuale e culturale, le sue posizioni politiche propense alla rivoluzione sociale, il sentimento provato per Modesta e, infine, malgrado la sua indole altruistica, nella relazione sessuale non riesce a superare quella scissione "tra il proprio piacere e quello della donna” (ibid.). Modesta, dopo il primo rapporto sessuale con Carlo, perplessa per non aver provato alcun piacere, pronuncia ad alta voce le stesse parole di Carmine: "Ma proprio non vi insegnano niente le vostre madri?", e rivolgendosi nel pensiero al suo primo amante: "Infatti, Carmine, non insegnano niente né a noi né a voi” (p. 163), sottolinea in primo luogo l'eccezionalità di Carmine e in secondo, aspetto più rilevante, punta su questa lacuna nell'educazione sessuale di entrambi i sessi. Anzi, l'unica fonte di informazioni sull'altro sesso, fonte alquanto artificiale, fuorviante e nociva, come nota Modesta, è la letteratura: "Adesso capisco: ti eri fatta una tua santa un po' dantesca da amare. O preferisci Petrarca, come credo? Allora hai fatto di me la tua pura e santa Laura. Poveri ragazzi! A noi Madame Bovary e a voi Laura" (p. 165). La cultura generata dai secoli della tradizione patriarcale contribuisce alla creazione di alcuni stereotipi che schiavizzano sia la donna sia l'uomo. Carlo li reiterava semplicemente, cosa che ammette lui stesso nelle parole: "Tu non lo sai, Modesta, che cosa sono gli uomini che ho conosciuto fin da piccolo, gli uomini che mi hanno formato. Non sai la loro solitudine, la loro ignoranza delle donne di cui credono di sapere tutto fin dalla prima prostituta da cui hanno avuto il coraggio d'andare" (p. 169). Carlo, giovane medico, di formazione positivistica e altruistica, si iscrive in "un'immagine che rende concretamente la rappresentazione del maschile come unico sog- 
getto desiderante, che segna» il mondo con il proprio desiderio e quindi unico soggetto tout court: «Desidero dunque sono»" (Ciccone, 2009, p. 85).

Il rapporto con la sessualità di entrambi gli amanti di Goliarda sembra essere riconducibile alla provenienza regionale di entrambi - Carmine siculo, Carlo lombardo - e con ciò agli stereotipi regionali di mascolinità, in sintonia con quanto propongono le narrazioni che stanno alla base delle costruzioni di identità nazionali ed etniche ${ }^{15}$. La costruzione di mascolinità geograficamente circoscritta si manifesta in primo luogo negli atteggiamenti e nelle prestazioni erotiche, cosa comprovata sia nella letteratura sia nella cinematografia. Come scrive Elena Dell'Agnese nella sezione intitolata Galanti per dna: stereotipi regionali di mascolinità: "Che il maschio del Sud sia più attraente e passionale del suo compatriota settentrionale è reso evidente in modo palese da molte opere cinematografiche" (Dell'Agnese, 2007, p. 18). La polarizzazione stereotipata del rapporto con la sessualità tra Carmine e Carlo va tuttavia oltre l'assegnazione a Carmine solo degli attributi di amante latino. Come nota Alberica Bazzoni,gli esempi di Carmine e Carlo dimostrano che sull'asse geografico ed etnico (Nord e Sud) vengono spostate le stereotipiche opposizioni tra mente (Carlo) e corpo (Carmine), tra razionalità e passione che sono tradizionalmente distribuite tra uomo e donna: "[...] rather than between men and women the distinction appears to be between Sicily and Northern Italy" (Bazzoni, 2018, p. 133).

\section{MASCHIO MAFIOSO}

Nel romanzo osserviamo una schiera di figure maschili che formano, a seconda della tappa della vita di Modesta, un importante punto di riferimento per la protagonista: la ispirano a uno sviluppo personale e l'assistono nella realizzazione dei suoi traguardi (Tuzzo, Mimmo, zio Jacopo, Matteo). Tra questi vi è un personaggio la cui costruzione romanzesca sembra derivare non solo dall'osservazione della società contemporanea

15 Le narrazioni nazionali rientrano nel campo di interesse della geopolitica critica cui si fa riferimento. Cf. Dell'Agnese, 2007, p. 5. 
e riferirsi alla cultura patriarcale in generale, ma provenire dai solchi del vissuto personale della scrittrice e della sua conoscenza dei lati più oscuri dell'isola natia.

Pietro è un vecchio servo della casa Brandiforti, fedele e discreto. Per anni si occupa con dedizione e riservatezza di Ippolito, figlio mongoloide della principessa Gaia e marito di Modesta per la quale comincia a riserbare una ossequiosa reverenza vedendo il suo abile e coraggioso atteggiamento nei confronti dell'uomo disabile, comunemente temuto e considerato ripugnante. La deferenza, la lealtà nonché una dedizione incondizionata segneranno da allora per sempre il suo rapporto con Modesta per la quale è pronto a fare tutto, anche uccidere "secondo la logica della vendetta che molto accoglie dalle metodiche mafiose [...]" (Fortini, 2011, p. 104). Infatti, Modesta è per Pietro molto di più di una padrona: è una matriarca ${ }^{16}$ potente, madre di una grande famiglia, composta da figli biologici adottivi, dai suoi/dalle sue amanti, da amici e servi, tutti però raccolti attorno a lei. Modesta crea attorno a sé un tipo di grande famiglia (collaborativa, leale, unita) all'interno della quale poteva nascere il pensare mafioso, che, come leggiamo nello studio di Dario Pietro Maugeri, "è un modo di essere ereditato e trasmesso in seno alla famiglia" (Maugeri, 2017, p. 2). Lo stesso studioso spiega, inoltre, che "la famiglia in cui origina e si perpetua il pensare mafioso è una famiglia di cultura materna" (ibid., p. 9) ovvero protettiva e dedita ma richiedente in cambio lealtà e ubbidienza nei confronti del genitore. I numerosi membri della non paradigmatica famiglia, fondata da Modesta non sul sangue e non sui legami biologici ma sulla reale cura, protezione e affetto, aderisce parzialmente a tale modello: parzialmente perché senza l'obbligo di obbedienza da parte dei membri della famiglia. Sia dunque la sua appartenenza alla cultura dell'isola, la sua prospettiva di chi, stando da sempre nei solchi della mentalità che non ammette altre soluzioni oltre la vendetta, la giustizia privata e la violenza, sia la lealtà

16 Sul mito del matriarcato in Sicilia cf. Corradini 1997, invece per approfondire il tema del rapporto di Modesta con le madri e di Modesta come madre si veda Di Rollo, 2016. 
nei confronti della padrona favoreggiano il pensare mafioso di Pietro che conserva e mette in atto dei concetti mafiosi, come dimostrato dal seguente dialogo:

- Dobbiamo considerarlo vendetta di famiglia, Mody?

- No, Pietro. Niente mutilazioni. Solo tre pallottole in mezzo agli occhi: una per Turi, una per Ciccio, una per Vincenzo. (p. 257)

Come si evince da questo scambio, Modesta sfrutta la natura mafiosa della servitù di Pietro essendo lei stessa ideatrice e promotrice della vendetta. In un altro momento sarà sempre lei a fermare, o meglio a guidare, la mano di Pietro prima di altri atti di giustizia privata da lui richiesti:

Ma uccidere Pasquale oggi sarebbe vendetta personale senza né capo né coda. [...] Noi come veri uomini e non femminucce isteriche facciamo finta di credere alla sua parziale fedeltà alla nostra idea, lo sfruttiamo, ce ne serviamo. [...] E quando verrà la buona stagione, non ti preoccupare che non mancheremo di dare una delusione a Pasquale che crede di comprarci per l'eternità per quattro favori che ci sta facendo. La nostra riconoscenza sarà una pallottola in mezzo agli occhi come è stato per Tudia e per gli altri, non ti preoccupare. (p. 273)

Il discorso riportato, nella sua forma maschile ("noi veri uomini"), per la crudeltà che vi abita ("la nostra riconoscenza sarà una pallottola"), per l'astuzia della fredda strategia che contiene ("e quando verrà la buona stagione") sembra essere pronunciato da un capo mafioso - che sarebbe questa volta Modesta stessa - rivolto a un suo gabellotto, per cui illustra perfettamente questa mascolinità, radicata nella tradizione dell'isola e alla quale anche la protagonista stessa partecipa attivamente ("noi veri uomini e non femminucce isteriche" ${ }^{17}$ ).

${ }_{17}$ Sull'identità di genere ambivalente di Modesta si veda Ross, 2012, pp. 234-241. 


\section{MASCHILITÀ IN COSTRUZIONE}

Il romanzo di Sapienza ci offre, inoltre, la possibilità di osservare la formazione della maschilità. La crescita di Eriprando, figlio di Carmine e Modesta, è rappresentata con grande intuito e conoscenza della psicologia maschile, tanto più apprezzabile in quanto il romanzo precede i più noti studi sulla maschilità ${ }^{18}$.

Quella di Prando si forma inizialmente in stretta relazione con la madre e con i valori da lei coltivati e trasmessi. Prando bambino cresce plasmato dalla mente di Modesta che gli trasferisce il suo profondo amore per la libertà e la sua ribellione contro tutte le forme di costrizione, schiavitù e ipocrisia. L'episodio che metaforizza questa educazione all'insegna della libertà personale dell'individuo è la liberazione di Prando dalle "fasce strette e dure per far crescere diritti e forti. Fasce rigide per educare, correggere, o anchilosare il corpo e la mente?" (p. 123).

Nel corso della lettura si nota che Prando crescendo palesa alcune caratteristiche della mascolinità tradizionale secondo i canoni del discorso patriarcale: autorevolezza, aggressività, anche se solo verbale, e propensione alla dominazione. Durante l'età giovanile diventa scontroso e provocatorio nei confronti di altri ragazzi e di Modesta stessa. A capire il suo comportamento ci viene in soccorso Franco La Cecla spiegando che nel periodo di passaggio all'età adulta, in assenza dei riti di passaggio tradizionali, i giovani ragazzi sono costretti a rompere da soli con l'infanzia per trovare la loro identità maschile. È dunque un periodo segnato da uno sforzo per differenziarsi e raggiungere un successo che sia diverso da quello della madre (La Cecla, 2014, pp. 118-119). Per potersi nominare maschi occorre, spiega La Cecla, liberarsi dall'influenza della madre. Nelle società tradizionali, i giovani dovevano essere separati dalle donne per cancellare dal proprio corpo l'influenza femminilizzante della madre e di altre donne nonché, in compagnia degli

18 I Men's Studies vedono il loro grande sviluppo soltanto a partire degli anni '80 e le opere più rinomate risalgono agli anni '80 e '90: Seidler, 1989; Connell, 1993; Badinter, 1992. 
uomini, assumere quello che La Cecla chiama "modi bruschi": durezza, impassibilità, scontrosità, aggressività (ibid., pp. 54-55). Infatti, come ricorda, citando Aristotele, Elisabeth Badinter, "È 1'uomo quello che genera l'uomo" (Badinter, 1993, p. 73) ovvero furono sempre gli uomini a formare durante i riti iniziatici la mascolinità socialmente accettata dei giovani ragazzi. Modesta intuendo questa necessità di Prando provoca lei stessa la rottura mandandolo a vivere da solo a Palermo, lontano dalla casa materna. La formazione della maschilità del giovane uomo si dovrà quindi compiere stimolata dagli altri maschi e secondo i canoni di genere previsti dalla società patriarcale che fu sicuramente quella siciliana degli anni Trenta.

La formazione di Prando va quindi in direzione della pratica della violenza e della prepotenza. Il ragazzo, come tanti della sua età, è attratto dal militarismo e dal combattimento che, secondo alcuni studiosi, è sempre stato l'unico modo per provare che si era diventati maschi (Tomasik, 2013, pp. 24-25). Dato il momento storico, il figlio di Modesta si sente attratto dall'ideologia fascista. Anche in questo delicato momento a moderare l'espandersi dell'influenza del militarismo fascista, viene in soccorso la saggezza di Modesta che spiega al figlio che la naturale voglia di confermare la propria maschilità nelle avventure spavalde può trovare sfogo in altri modi, anche fuori dai contesti militari e, cosa più importante, preservando la propria libertà e indipendenza.

Il tuo desiderio d'avventura mi ha innervosito. Comprati la macchina che volevi e torna a gareggiare coi maschi come te, o parti per l'America, ruba, insomma, fai quello che vuoi! ma che tutto nasca da te e non da un ordine del re, del Duce, o del Führer! Desiderare la guerra è già piegare il futuro, e non solo il tuo, verso la sventura. Lo vuoi capire sì o no? È l'ultima volta che cerco di farmi capire da te e dai maschi boriosi come te. Tu non appartieni allo Stato, né a me, e non ti illudere che io dia ordini. Sangue di Giuda! Ma come si deve far per farvi capire che molti desideri vi vengono inculcati dall'alto per usarvi? (p. 379)

Nonostante la saggezza di Modesta e nonostante i suoi metodi educativi che aspirano alla crescita nel rispetto della libertà e della democrazia, nonostante, infine, lei stessa rappresenti un'identità femminile d'avanguardia, Modesta non riesce a formare un'identità maschile alla 
pari. Prando, dopo la rottura con la femminilità della casa familiare, non si staccherà mai dalla mascolinità tradizionale che lo ha in seguito plasmato, innestandogli caratteristiche quali la tendenza al predominio e talvolta anche il despotismo. Modesta lo coglie nelle parole metaforiche: "Tu sei vecchio quanto il potere di quest'isola, e sei bello anche, della bellezza vecchia di quest'isola. Mi piace guardarti. Mi ricordi un vecchio astuto come il mare e calmo come il Monte che mi incantava da ragazzina" (p. 476).

\section{CONCLUSIONI}

Le più importanti figure maschili nel romanzo di Goliarda Sapienza formano una struttura letteraria ben ponderata, anzi quasi geometrica: essi si potrebbero distribuire nei riquadri prodotti dall'incrocio di due assi del sistema di coordinate: l'asse che va dal simbolico al reale e l'asse che va dal locale all'universale. Il padre e il marito della protagonista appaiono nel romanzo come figure universali della patriarcalità ma al contempo simboleggiano il ribaltamento dell'ordine patriarcale auspicato dalla scrittrice. I due amanti, Carmine e Carlo, sono attuazioni reali della paternità e del ruolo maritale. Essi rappresentano inoltre due diversi approcci alla sessualità maschile associabili agli stereotipi geopolitici, quelli del Nord e del Sud. Si collocano quindi nel riquadro formato dai vettori del reale e del locale. Una rappresentazione dell'ambigua mascolinità siciliana (locale), segnata dal pensiero mafioso è quella rappresentata da Pietro, leale, fedele ma spietato per chi è nemico della famiglia. È al contempo una figura simbolica atta a valere le antiche e immutabili leggi della Sicilia, "il suo oscuro profondo" (p. 273), il suo fascino a cui è difficile sottrarsi. Lo suggerisce Modesta pensando: "Lo devo guardare negli occhi, non si sfugge al cerchio del suo sguardo, né attraversando il mare, come dice lui, né fissando fuori dal finestrino di un treno le distese sterminate di boschi e colture ordinate, allineate artificiosamente senza avventura [...]" (p. 274). È invece molto reale ma con una valenza universale la rappresentazione della maschilità in progress, del formarsi di un'identità maschile, con le caratteristiche mascoline risalenti alla cultura patriarcale, osservata e illustrata da Prando. 
Goliarda Sapienza crea quindi un sistema (uno schema) molto preciso per esprimere le sue idee. I tre personaggi con una valenza simbolica (il padre, il marito, Pietro) rappresentano le leggi arcaiche, rispettivamente universali e locali (siciliane), di prepotenza e di violenza maschili. Tuttavia, mentre le prime sono simbolicamente trasgredite (l'anonimo padre scompare nel nulla e il marito è un disabile mentale) le seconde rimangono valide e sono praticate. I tre personaggi con una valenza reale (Carmine, Carlo, Prando) rappresentano esempi concreti della crescita e dell'attuazione dei ruoli maschili nei contesti geograficamente circoscritti (Carmine/Sud, Carlo/Nord) e in un percorso evolutivo universale (Prando).

Tale sistema dei personaggi - ben equilibrato e compatto - è inoltre prova delle capacità scrittorie di Sapienza e della sua consapevolezza della letteratura, tanto più che lo stesso sistema di coordinate (simbolico-reale, locale-universale) potrebbe essere applicato, sempre all'avviso di chi scrive, agli altri piani della struttura romanzesca (narrativo, linguistico, semantico), cosa che costituisce campo per un'ulteriore ricerca.

\section{BIBLIOGRAFIA}

Badinter, E. (1993). XY tożsamość mężczyzny (G. Przewłocki, Trans.). Warszawa: W.A.B (Original work, X Y. De l'identité masculine, published 1992).

Bazzoni, A. (2012). Gli anni e le stagioni: prospettive su femminismo, politica e storia ne "L'arte della gioia". In G. Providenti (Ed.), "Quel sogno d'essere" di Goliarda Sapienza. Percorsi critici su una delle maggiori autrici del Novecento italiano (pp. 33-52). Roma: Aracne.

Bazzoni, A. (2018). Writing for Freedom. Body, Identity and Power in Goliarda Sapienza's Narrative. Berna: Peter Lang.

Bazzoni, A., Bond, E., \& Wehling-Giorgi, K. (Eds.). (2016). Goliarda Sapienza in Context. Intertextual Relationships With Italian And European Culture. Vancouver: Fairleigh Dickinson University Press.

Ceccatty de, R. (2005, settembre, 15). Sapienza, princesse hérétique. Le Monde. Retrieved from https://www.lemonde.fr/livres/article/2005/09/15/sapienza-princesse-heretique_689173_3260.html. 
Ciccone, S. (2009). Essere maschi. Tra potere e libertà. Torino: Rosenberg $\&$ Sellier.

Connell, R. W. (1995). Masculinities. Berkeley and Los Angeles: California Press.

Corradini, A. M. (1997). Meteres. Il Mito Del Matriarcato In Sicilia. Enna: Papiro.

De Biasio, A. (2010). Studiare il maschile. Allegoria. Per uno studio materialistico della letteratura, 61(1), 9-36. Retrieved from https://www.torrossa.com/it/resources/an/2512827.

Dell'Agnese, E. (2007). Tu vuo' fa' l'americano: la costruzione della mascolinità nella geopolitica popolare italiana. In E. Dell'Agnese, \& E. Ruspini (Eds.), Mascolinità all'italiana. Costruzioni, narrazioni, mutamenti (pp. 3-34). Torino: Utet Libreria.

Devoto, G., \& Oli, G. C. (1990). Dizionario della lingua italiana. Firenze: Le Monnier.

Di Rollo, A. (2016). Reforging the Maternal Bond: Motherhood, MotherDaughter Relationship, and Female Relationality in Goliarda Sapienza's "L'arte della gioia". In A. Bazzoni, E. Bond, \& K. Wehling-Giorgi (Eds.). (2016). Goliarda sapienza in context. Intertextual Relationships With Italian And European Culture (pp. 33-47). Vancouver: Fairleigh Dickinson University Press.

Farnetti, M. (2011). "L'arte della gioia" e il genio dell'omicidio. In M. Farnetti (Ed.), Appassionata Sapienza (pp. 89-100). Milano: La Tartaruga.

Fortini, M., \& Bono, P. (Eds.). (2007). Il romanzo del divenire. Un Bildungsroman delle donne? Pavona: Iacobelli.

Fortini, L. (2011). "L'arte della gioia" e il genio dell'omicidio mancato. In M. Farnetti (Ed.), Appassionata Sapienza (pp. 101-126). Milano: La Tartaruga.

La Cecla, F. (2014). Szorstkim być (H. Serkowska, Trans.).Warszawa: Sic! (Original work, Modi bruschi. Antropologia del maschio, published 2000).

Maugeri, D. P. (2017). Sociologia della mafia: paradigmi incerti visti da dentro. Retrieved from https://www.diritto.it/sociologia-della-mafia-paradigmi-incerti-visti-dentro/.

Mazzanti, R., Neonato, S., \& Sarasini, B. (Eds.). (2016). L'invenzione delle personagge, Roma: Iacobellieditore. 
Michelacci, L. (2015). La storia come trasgressione. "L'arte della gioia" di Goliarda Sapienza. Griseldaonline, 15, 1-13. Retrieved from https:// griseldaonline.unibo.it/article/view/9141/9021.

Providenti, G. (2010). La porta è aperta. Vita di Goliarda Sapienza. Catania: Villaggio Maori.

Providenti, G. (Ed.). (2012). "Quel sogno d'essere" di Goliarda Sapienza. Percorsi critici su una delle maggiori autrici del Novecento italiano. Roma: Aracne.

Providenti, G. (2016). Goliarda Sapienza. La porta della gioia. Roma: Nova Delphi Libri.

Rizzarelli, M. (2018). Goliarda Sapienza. Gli spazi della libertà, il tempo della gioia. Roma: Carocci.

Ross, Ch. (2012). Identità di genere e sessualità nelle opere di Goliarda Sapienza: finzioni necessariamente queer. In G. Providenti (Ed.), "Quel sogno d'essere" di Goliarda Sapienza. Percorsi critici su una delle maggiori autrici del Novecento italiano (pp. 223-242). Roma: Aracne.

Rotondo, L. (2011). L'arte di una vita. In M. Farnetti (Ed.), Appassionata Sapienza (pp. 25-30). Milano: La Tartaruga.

Sapienza, G. (2005). L’art de la joie (N. Castagné, Trans.). Paris: Éditions Viviane Hamy.

Sapienza, G. (2014). L'arte della gioia. Torino: Einaudi.

Sapienza, G. (2018). Sztuka radości (T. Kwiecień, Trans.). Białystok: Wydawnictwo Kobiece.

Scarfone, G. (2018). Goliarda Sapienza. Un'autrice ai margini del sistema letterario. Massa: Transeuropa.

Seidler, V. J. (1989). Rediscovering Masculinity: Reason, Language, and Sexuality. New York: Routledge,

Tomasik, T. (2013). Wojna-męskość - literatura. Słupsk: Wydawnictwo Naukowe Akademii Pomorskiej w Słupsku.

Trevisan, A. (2016). Goliarda Sapienza: una voce intertestuale (1996-2016). Milano: La Vita Felice.

Vigorita, M. (2011). Linee per un ritratto. In M. Farnetti (Ed.), Appassionata Sapienza (pp. 15-24). Milano: La Tartaruga.

Riassunto: Lo scopo dell'articolo è quello di analizzare - ricorrendo all'approccio strutturalista il sistema dei personaggi maschili ne L'arte della gioia di Goliarda Sapienza e il significato universale o locale, simbolico o reale - attribuitogli dalla scrittrice, sull'esempio di sei personaggi principali. Tale approccio consente di addentrarsi nel testo letterario lasciando al di fuori dell'analisi 
il contesto biografico, storico ed ideologico, e di focalizzarsi sulle dinamiche interne puramente letterarie. Il padre e il marito di Modesta, in quanto figure universali del sistema patriarcale, illustrano e simboleggiano la sovversione delle sue leggi alla quale contribuisce Modesta. I suoi amanti, Carmino e Carlo, sono modelli di una reale attuazione degli importanti ruoli maschili (padre, marito, amante), la cui rappresentazione è tuttavia limitata dagli stereotipi geopolitici. Pietro, il leale gabellotto di Modesta, incarna una relazione interpersonale tipicamente locale, siciliana ed in quanto tale simboleggia la persistenza di alcune arcaiche leggi della società siciliana. Con Prando, figlio di Modesta, Sapienza illustra realisticamente il passaggio dall'adolescenza alla mascolinità, mostrando alcune leggi universali su cui si basa tale passaggio. Il sistema dei personaggi maschili risulta essere una struttura geometrica e molto chiara, cosa che testimonia della grande consapevolezza della letteratura della scrittrice nonché delle sue capacità scrittorie.

Parole chiave: Goliarda Sapienza, Arte della gioia, mascolinità, universalità, sicilianità 